\title{
Differential equations for the cuspoid canonical integrals
}

J. N. L. Connor

A. A. Noyes Laboratory of Chemical Physics, California Institute of Technology, Pasadena, California 91125 and Department of Chemistry, University of Manchester, Manchester M13 9PL, England ${ }^{\text {a) }}$

P. R. Curtis

Department of Chemistry, University of Manchester, Manchester M13 9PL, England ${ }^{\text {b) }}$

(Received 23 November 1983; accepted for publication 2 March 1984)

Differential equations satisfied by the cuspoid canonical integrals $I_{n}(\mathbf{a})$ are obtained for arbitrary values of $n \geqslant 2$, where $n-1$ is the codimension of the singularity and $\mathbf{a}=\left(a_{1}, a_{2}, \ldots, a_{n-1}\right)$. A set of linear coupled ordinary differential equations is derived for each step in the sequence $I_{n}(0,0, \ldots, 0,0)$ $\rightarrow I_{n}\left(0,0, \ldots, 0, a_{n-1}\right) \mapsto I_{n}\left(0,0, \ldots, a_{n-2}, a_{n-1}\right) \rightarrow \ldots \rightarrow I_{n}\left(0, a_{2}, \ldots, a_{n-2}, a_{n-1}\right) \rightarrow I_{n}\left(a_{1}, a_{2}, \ldots, a_{n-2}, a_{n-1}\right)$. The initial conditions for a given step are obtained from the solutions of the previous step. As examples of the formalism, the differential equations for $n=2$ (fold), $n=3$ (cusp), $n=4$ (swallowtail), and $n=5$ (butterfly) are given explicitly. In addition, iterative and algebraic methods are described for determining the parameters $a$ that are required in the uniform asymptotic cuspoid approximation for oscillating integrals with many coalescing saddle points. The results in this paper unify and generalize previous researches on the properties of the cuspoid canonical integrals and their partial derivatives.

PACS numbers: 02.30.Hq, 02.30.Bi, 03.80. $+\mathrm{r}$

\section{INTRODUCTION}

There has been considerable interest recently in the properties of the cuspoid canonical integrals $I_{n}(\mathbf{a}) \cdot{ }^{1-9}$ The integral $I_{n}(\mathbf{a})$ is defined by ${ }^{2,10}$

$$
\begin{aligned}
I_{n}\left(a_{1}, a_{2}, \ldots, a_{n-1}\right)= & \int_{-\infty}^{\infty} \exp \left[i \left(a_{1} u+a_{2} u^{2}+\cdots\right.\right. \\
& \left.\left.+a_{n-1} u^{n-1}+u^{n+1}\right)\right] d u,
\end{aligned}
$$

where the coefficients $a_{j}$ are real and $n$ is a positive integer with $n \geqslant 2$. The name cuspoid canonical integral comes from catastrophe theory, where the polynomial in the exponent of Eq. (1.1) is the canonical form for the cuspoid catastrophes, with $n-1$ the codimension of the singularity. ${ }^{10-13}$

The integral $I_{n}(\mathbf{a})$, together with its partial derivatives $\partial I_{n} / \partial a_{1}, \partial I_{n} / \partial a_{2}, \ldots, \partial I_{n} / \partial a_{n-1}$ occur in the asymptotic analysis of many short wavelength scattering theories involving atoms, molecules, and nuclear heavy ions as well as in acoustic, electromagnetic, and water-wave propagation, and in other problems. References to a large number of relevant papers can be found in Refs. 4, 6, and 9 .

We have recently investigated ${ }^{4-6,8,9}$ the differential equations satisfied by $I_{n}(\mathbf{a})$ and its partial derivatives for the special cases of $n=2$ (fold), $n=3$ (cusp), and $n=4$ (swallowtail). For $n=2$, Eq. (1.1) is proportional to Airy's inte$\operatorname{gral}^{14} \mathrm{Ai}(x)$ and the differential equation for $\mathrm{Ai}(x)$ is well known. ${ }^{15}$ When $n=3$, Eq. (1.1) is identical with Pearcey's integral $P(x, y) .{ }^{16-18}$ The differential equations for $P(x, y)$ were first derived by Pearcey ${ }^{17,18}$ and used by him to numerically evaluate $P(x, y)$. For the swallowtail canonical integral $(n=4)$, which is also written $S(x, y, z)$, the relevant differential equations have been obtained by ourselves and Farrelly ${ }^{8}$ and used in numerical computations. ${ }^{8,9}$

The purpose of the present paper is to derive the differential equations satisfied by the cuspoid canonical integrals

\footnotetext{
${ }^{\text {a) }}$ Permanent address.

${ }^{b}$ Present address: Marconi Space and Defense Systems Ltd., The Grove, Warren Lane, Stanmore, Middlesex HA7 4LY, England.
}

for any value of $n$. Our treatment generalizes and unifies the previous work $^{4-6,8,9,15,17,18}$ in which each value of $n=2,3,4$ was treated as a separate case.

We derive the differential equations in Sec. II. The basic idea is to obtain a set of linear coupled ordinary differential equations for each step in the sequence $I_{n}(0,0, \ldots, 0,0)$ $\rightarrow I_{n}\left(0,0, \ldots, 0, a_{n-1}\right) \rightarrow I_{n}\left(0,0, \ldots, a_{n-2}, a_{n-1}\right) \rightarrow, \ldots$, $\rightarrow I_{n}\left(0, a_{2}, \ldots, a_{n-2}, a_{n-1}\right) \rightarrow I_{n}\left(a_{1}, a_{2}, \ldots, a_{n-2}, a_{n-1}\right)$. The solutions of the differential equations for a given step provide the initial conditions for the next step, with the initial conditions at the origin $(0,0, \ldots, 0,0)$ being obtained directly from Eq. (1.1). An advantage of this scheme is that it allows the numerical evaluation of the derivatives $\partial I_{n} / \partial a_{1}$, $\partial I_{n} / \partial a_{2}, \ldots, \partial I_{n} / \partial a_{n-1}$ as well as $I_{n}$ at a given value of $\mathbf{a}$.

Section III provides concrete examples of our formalism. We show for the special cases of $n=2,3,4$ that our equations are equivalent to those obtained earlier. We also give the differential equations for the butterfly canonical integral $(n=5)$, as they have not been reported in the literature before.

An important use of $I_{n}$ and $\partial I_{n} / \partial a_{1}$, $\partial I_{n} / \partial a_{2}, \ldots, \partial I_{n} / \partial a_{n-1}$ is in uniform asymptotic approximations for one-dimensional oscillating integrals with many nearly coincident stationary phase or saddle points. ${ }^{1,2,10,19-23}$ In Section IV we show how the iterative and algebraic methods of Ref. 9 for calculating the parameters a that are required in $I_{n}$ and its partial derivatives can be generalized from the case of $n=4$ to the case of arbitrary $n$. Thus the techniques reported in this paper allow in principle the application of uniform asymptotic approximations for integrals with an arbitrary number of nearly coincident saddle points. Our conclusions are in Sec. V.

\section{DIFFERENTIAL EQUATIONS}

The integral representation (1.1) for $I_{n}(\mathbf{a})$ is conditionally convergent. This has the disadvantage that repeated differentiation under the integral sign eventually produces a 
divergent integral. ${ }^{8,24}$ To avoid this problem, we deform the contour of integration $C_{n}$ so that it starts and ends in sectors of the complex $u$ plane where the integrand is exponentially small. We can write

$$
I_{n}(\mathbf{a})=\int_{C_{n}} \exp \left[i P_{n}(u)\right] d u,
$$

with

$$
P_{n}(u)=u^{n+1}+\sum_{j=1}^{n-1} a_{j} u^{j}
$$

When $n$ is an even integer, we choose $C_{n}$ to start on the ray $\arg u=\pi-\frac{1}{2} \pi /(n+1)$ and finish on the ray $\arg u=\frac{1}{2} \pi /$ $(n+1)$. For $n$ odd, the contour $\arg u=\pi+\frac{1}{2} \pi /(n+1)$ to $\arg u=\frac{1}{2} \pi /(n+1)$ is an appropriate choice for $C_{n}$.

We now wish to derive a set of differential equations for the integration $I_{n}(0,0, \ldots, 0,0) \rightarrow I_{n}\left(a_{1}, a_{2}, \ldots, a_{n-2}, a_{n-1}\right)$. To accomplish this we use a sequence of steps $I_{n}(0,0, \ldots, 0,0)$ $\rightarrow I_{n}\left(0,0, \ldots, 0, a_{n-1}\right) \rightarrow I_{n}\left(0,0, \ldots, a_{n-2}, a_{n-1}\right)$

$\rightarrow, \ldots, \rightarrow I_{n}\left(0, a_{2}, \ldots, a_{n-2}, a_{n-1}\right) \rightarrow I_{n}\left(a_{1}, a_{2}, \ldots, a_{n-2}, a_{n-1}\right)$, such that in each step $k$ all the $a_{j}$ are constant except for $a_{k}$.

We derive the differential equation for the variable $a_{1}$ first. From Eq. (2.2) we have

$$
P_{n}^{\prime}(u)=(n+1) u^{n}+\sum_{j=1}^{n-1} j a_{j} u^{j-1} .
$$

Now partial differentiation of Eq. (2.1) with respect to $a_{1}$ is equivalent to multiplication of the integrand by $i u$. It then follows from Bleistein ${ }^{20}$ that $I_{n}(\mathbf{a})$ satisfies the differential equation

$$
P_{n}^{\prime}\left(-i \frac{\partial}{\partial a_{1}}\right) I_{n}\left(a_{1}, a_{2}, \ldots, a_{n-1}\right)=0,
$$

because

$$
\int_{C_{n}} P_{n}^{\prime}(u) \exp \left[i P_{n}(u)\right] d u=0 .
$$

The operator in Eq. (2.4) is given explicitly by

$$
\begin{aligned}
P_{n}^{\prime}\left(-i \frac{\partial}{\partial a_{1}}\right)= & (-i)^{n}(n+1) \frac{\partial^{n}}{\partial a_{1}^{n}} \\
& +\sum_{j=1}^{n-1}(-i)^{j-1} j a_{j} \frac{\partial^{j-1}}{\partial a_{1}^{j-1}} .
\end{aligned}
$$

We then obtain from Eq. (2.4) the differential equation for $I_{n}(\mathbf{a})$ in the variable $a_{1}$ :

$$
\frac{\partial^{n} I_{n}}{\partial a_{1}^{n}}+\sum_{j=1}^{n-1}(-i)^{j-n-1} \frac{j}{n+1} a_{j} \frac{\partial^{j-1} I_{n}}{\partial a_{1}^{j-1}}=0
$$

To proceed further we introduce the functions $I_{n}^{(p)}(\mathbf{a})$ defined by

$$
I_{n}^{(p)}(\mathbf{a})=\frac{\partial^{p} I_{n}(\mathbf{a})}{\partial a_{1}^{p}}, \quad p=0,1, \ldots
$$

with the convention that

$$
I_{n}^{(0)}(\mathbf{a}) \equiv I_{n}(\mathbf{a})
$$

and

$$
I_{n}^{(p)}(\mathbf{a}) \equiv 0 \quad \text { when } p<0 .
$$

For $n=4$ (swallowtail canonical integral), $I_{4}^{(1)}$ and $I_{4}^{(2)}$ are the same as the functions $H$ and $G$, respectively, that were introduced in Ref. 8. Our strategy now is to derive sets of coupled linear ordinary differential equations for each of the variables $a_{2}, a_{3}, \ldots, a_{n-1}$ in which the $I_{n}^{(p)}$ are treated as independent functions.

Differentiating Eq. (2.6) $p$ times with respect to $a_{1}$ with $p=0,1, \ldots, n-2$ gives

$$
\begin{aligned}
& \frac{\partial^{n} I_{n}^{(p)}}{\partial a_{1}^{n}}+\sum_{j=1}^{n-1}(-i)^{j-n-1} \frac{j}{n+1} a_{j} \frac{\partial^{j-1} I_{n}^{(p)}}{\partial a_{1}^{j-1}} \\
& \quad+(-i)^{-n} \frac{p}{n+1} I_{n}^{(p-1)}=0, \quad p=0,1, \ldots, n-2
\end{aligned}
$$

Next we use differential identities satisfied by $I_{n}^{(p)}$ to change the integration variable from $a_{1}$ to the variable $a_{j}$, $j=2,3, \ldots, n-1$.

From the integral representation $(2.1)$ for $I_{n}(a)$ we deduce that

$$
I_{n}^{(p)}(\mathbf{a})=i^{p} \int_{C_{n}} u^{p} \exp \left[i P_{n}(u)\right] d u, \quad p=0,1, \ldots
$$

and differentiation of this equation $q$ times $(q=0,1,2, \ldots)$ with respect to $a_{j}$ gives

$$
\begin{aligned}
& \frac{\partial^{q} I_{n}^{(p)}}{\partial a_{j}^{q}}=i^{p+q} \int_{C_{n}} u^{p+j q} \exp \left[i P_{n}(u)\right] d u, \\
& p, q=0,1, \ldots, j=1,2, \ldots, n-1 .
\end{aligned}
$$

If we change labels $p \rightarrow r, q \rightarrow s, j \rightarrow l$ in Eq. (2.10) we have

$$
\begin{aligned}
& \frac{\partial^{s} I_{n}^{(r)}}{\partial a_{l}^{s}}=i^{r+s} \int_{C_{n}} u^{r+l s} \exp \left[i P_{n}(u)\right] d u, \\
& r, s=0,1, \ldots, l=1,2, \ldots, n-1 .
\end{aligned}
$$

It is now clear that if $r$ is chosen to be

$$
r=p+j q-l s,
$$

then we obtain from Eqs. (2.10) and (2.11) the identity

$$
\frac{\partial^{q} I_{n}^{(p)}}{\partial a_{j}^{q}}=i^{q(1-j)-s(1-l)} \frac{\partial^{s} I_{n}^{(p+j q-t s)}}{\partial a_{l}^{s}}, \begin{aligned}
& p, q, s=0,1, \ldots, \\
& j, l=1,2, \ldots, n-1 .
\end{aligned}
$$

This relation lets us change from the variable $a_{j}$ on the lefthand side to the variable $a_{l}$ on the right-hand side. For example for the case $j=1$ required below we have

$$
\frac{\partial^{q} I_{n}^{(p)}}{\partial a_{1}^{q}}=i^{s(l-1)} \frac{\partial^{s} I_{n}^{(p+q-l s)}}{\partial a_{l}^{s}}, \quad \begin{aligned}
& p, q, s=0,1, \ldots, \\
& l=1,2, \ldots, n-1 .
\end{aligned}
$$

The relation (2.12) encompasses a large number of individual identities. For the swallowtail case $n=4$ some of them are reported in Ref. 8.

We next return to the differential equation $(2.8)$ for $I_{n}^{(p)}$ and set

$$
a_{j}=0 \text { for } j<l \text { with } l=1,2, \ldots, n-1 \text {. }
$$

In addition for each $l$ we only keep terms which have $p=0,1, \ldots, l-1$. We obtain

$$
\begin{aligned}
& \frac{\partial^{n} I_{n}^{(p)}}{\partial a_{1}^{n}}+\sum_{j=l}^{n-1}(-i)^{j-n-1} \frac{j}{n+1} a_{j} \frac{\partial^{j-1} I_{n}^{(p)}}{\partial a_{1}^{j-1}} \\
& \quad+(-i)^{-n} \frac{p}{n+1} I_{n}^{(p-1)}=0, \\
& \quad a_{j}=0 \text { for } j<l, p=0,1, \ldots, l-1, l=1,2, \ldots, n-1 .
\end{aligned}
$$


We next wish to convert Eq. (2.15) into a set of differential equations with respect to $a_{l}$ using the identity (2.13). However, inspection of Eq. (2.13) shows that for fixed $p, q, l$ there is still a choice to be made for $s$. We choose $s$ so that the superscript $k=p+q-l s$ on the right-hand side of Eq. (2.13) is zero or is the smallest possible integer for a given $p, q, l$. We choose $s$ in this way because it introduces the minimum number of independent functions $I_{n}^{(k)}$ into Eq. (2.15) when the identity (2.13) is used. Our choice for $s$ is automatically satisfied if for a given $p, q, l$ we calculate it from the equation

$$
s_{q}=\operatorname{int}[(p+q) / l] \text {, }
$$

where int $=$ integer part of. Note that $s_{q}$ is also a function of $p$ and $l$, but this has not been indicated explicitly in Eq. (2.16) in order to simplify the notation.

With this choice for $s$, Eq. (2.13) becomes for $q=n$

$$
\frac{\partial^{n} I_{n}^{(p)}}{\partial a_{1}^{n}}=l^{s_{n}(l-1)} \frac{\partial^{s_{n}} I_{n}^{\left(p+n-i s_{n}\right)}}{\partial a_{l}^{s_{n}}}, \begin{aligned}
& p=0,1, \ldots, \\
& l=1,2, \ldots, n-1,
\end{aligned}
$$

where

$$
s_{n}=\operatorname{int}\left(\frac{p+n}{l}\right) .
$$

We now use the identity $(2.17)$ to change variable from $a_{1}$ to $a_{1}$ in Eq. (2.15) obtaining

$$
\begin{aligned}
& \frac{\partial^{s_{n}} I_{n}^{\left(n+p-l s_{n}\right)}}{\partial a_{l}^{s_{n}}}+\sum_{j=l}^{n-1} i^{\left(s_{j-1}-s_{n}\right)(l-1)+n+1-j} \frac{j}{n+1} \\
& \times a_{j} \frac{\partial^{s_{j}-1} I_{n}^{\left(j-1+p-l_{s_{j-1}}\right)}}{\partial a_{l}^{s_{j-1}}} \\
& +i^{n-s_{n}(l-1)} \frac{p}{n+1} I_{n}^{(p-1)}=0
\end{aligned}
$$

$a_{j}=0$ for $j<l, p=0,1, \ldots, l-1, l=1,2, \ldots, n-1$,

and

$$
s_{j-1}=\operatorname{int}[(p+j-1) / l] .
$$

Equation (2.19) is the main result of this paper. It represents $n-1$ sets of coupled ordinary linear differential equations. Each set involves only a single variable $a_{l}$ with the condition $a_{j}=0$ for $j<l$. For each value of $l$, there are $l$ equations $(p=0,1, \ldots, l-1)$ which contain $l$ independent functions $I_{n}^{(p)}$.

Equation (2.19) represents sufficient equations to evaluate $I_{n}$ and $\partial I_{n} / \partial a_{1}, \partial I_{n} / \partial a_{2}, \ldots, \partial I_{n} / \partial a_{n-1}$ at any point $\left(a_{1}, a_{2}, \ldots, a_{n-1}\right)$. The procedure to accomplish this is as follows

(a) Set $l=n-1$ in Eq. (2.19). This gives a set of $n-1$ differential equations in the variable $a_{n-1}$ with $a_{1}=a_{2}=\cdots=a_{n-2}=0$.

(b) Evaluate $I_{n}^{(p)}$ at the origin $(0,0, \ldots, 0)$ for all required values of $p=0,1, \ldots, n-1$ (see below). This supplies all the necessary initial conditions at the origin for Eq. (2.19) with $l=n-1$.

(c) Integrate numerically from $(0,0, \ldots, 0,0)$ to $\left(0,0, \ldots, 0, a_{n-1}\right)$ using a standard integration routine for coupled ordinary linear differential equations.

(d) Set $l=n-2$ in Eq. (2.19). This gives a set of $n-2$ differential equations in the variable $a_{n-2}$ with $a_{j}=0$ for $j<n-2$. (e) Use the identity (2.12) to transform the solutions at $\left(0,0, \ldots, 0, a_{n-1}\right)$ obtained in step (c) into initial conditions for Eq. (2.19) with $l=n-2$ [step (d)].

(f) Integrate from $\left(0,0, \ldots, 0,0, a_{n-1}\right)$ to $\left(0,0, \ldots, 0, a_{n-2}, a_{n-1}\right)$.

(g) Repeat steps (d), (e), (f) for $l=n-3, n-4, \ldots, 3,2$. We have now reached the point $\left(0, a_{2}, a_{3}, \ldots, a_{n-2}, a_{n-1}\right)$.

(h) Finally set $l=1$ and integrate Eq. (2.19) [which is now equivalent to Eq. (2.6)] from $\left(0, a_{2}, \ldots, a_{n-1}\right)$ to $\left(a_{1}, a_{2}, \ldots, a_{n-1}\right)$ using as initial conditions the solutions from the previous integration step together with Eq. (2.12). At $\left(a_{1}, a_{2}, \ldots, a_{n-1}\right)$ we have now evaluated $I_{n}$. Its derivatives $\partial I_{n} / \partial a_{1}, \partial I_{n} / \partial a_{2}, \ldots, \partial I_{n} / \partial a_{n-1}$ are obtained from the solutions of Eq. (2.19) together with the identity (2.17).

In step (b), it is necessary to calculate the initial conditions at the origin $(0,0, \ldots, 0)$ for Eq. (2.19) with $l=n-1$. The allowed values of $p$ in this case are $p=0,1, \ldots, n-2$. If we evaluate the superscripts $s_{n}, n+p-(n-1) s_{n}, s_{n-2}$, and $n-2+p-(n-1) s_{n-2}$ in Eq. (2.19) then it is found that the set of differential equations for $l=n-1$ consists of terms of the form

$$
\frac{\partial I_{n}^{(p)}}{\partial a_{n-1}}, \quad p=0,1, \ldots, n-2 \text { and } \frac{\partial^{2} I_{n}^{(0)}}{\partial a_{n-1}^{2}},
$$

where the term of second order comes from $p=n-2$. The initial conditions at the origin that we must calculate are therefore, of the form

$$
I_{n}^{(p)}, \quad p=0,1, \ldots, n-2 \text { and } \frac{\partial I_{n}^{(0)}}{\partial a_{n-1}} .
$$

However Eqs. (2.1) and (2.9) show that

$$
\frac{\partial I_{n}^{(0)}}{\partial a_{n-1}}=-i^{-n} I_{n}^{(n-1)} .
$$

Hence, we can fix the initial conditions at the origin for Eq. (2.19) with $l=n-1$ provided we can calculate

$$
I_{n}^{(p)}(0,0, \ldots, 0), \quad p=0,1, \ldots, n-1 .
$$

Now Eq. (2.9) shows that

$$
\begin{aligned}
& I_{n}^{(p)}(0,0, \ldots, 0) \\
& \quad=i^{p} \int_{-\infty}^{\infty} u^{p} \exp \left(i u^{n+1}\right) d u, \quad p=0,1, \ldots, n-1,
\end{aligned}
$$

which can be written in the alternative form

$$
\begin{aligned}
I_{n}^{(p)}(0,0, \ldots, 0)= & i^{p} \int_{0}^{\infty} u^{p} \exp \left(i u^{n+1}\right) d u \\
& +(-1)^{p} i^{p} \int_{0}^{\infty} u^{p} \exp \left(i(-1)^{n+1} u^{n+1}\right) d u, \\
& p=0,1, \ldots, n-1 .
\end{aligned}
$$

The integrals in Eq. (2.26) can now be evaluated with the help of the results ${ }^{25,26}$

$$
\begin{aligned}
& \int_{0}^{\infty} u^{p} \exp \left(i u^{n+1}\right) d u \\
& =\frac{1}{n+1} \Gamma\left(\frac{p+1}{n+1}\right) \exp \left[i \frac{\pi}{2}\left[\frac{p+1}{n+1}\right]\right], \\
& p=0,1, \ldots, n-1,
\end{aligned}
$$

and 


$$
\begin{aligned}
\int_{0}^{\infty} u^{p} \exp \left(-i u^{n+1}\right) d u \\
\quad=\frac{1}{n+1} \Gamma\left[\frac{p+1}{n+1}\right] \exp \left[-i \frac{\pi}{2}\left[\frac{p+1}{n+1}\right]\right] . \\
p=0,1, \ldots, n-1 .
\end{aligned}
$$

Consider first the case $n=2 m=$ even positive integer. Applying Eqs. (2.27) and (2.28) to Eq. (2.26) shows that

$$
\begin{aligned}
& I_{2 m}^{(p)}(0,0, \ldots, 0) \\
& \quad=\frac{2}{2 m+1} \Gamma\left[\frac{p+1}{2 m+1}\right] \cos \left[\frac{\pi}{2}\left[\frac{p+1}{2 m+1}+p\right]\right], \\
& m=1,2, \ldots, p=0,1, \ldots, 2 m-1 .
\end{aligned}
$$

Next consider the case $n=2 m+1=$ odd positive integer. If $p=2 q+1=$ odd positive integer, then the integrand of Eq. (2.25) is an odd function of $u$ and

$$
I_{2 m+1}^{(2 q+1)}(0,0, \ldots, 0)=0, \quad m=1,2, \ldots, q=0,1, \ldots, m-1 .
$$

If however, $p=2 q=$ even integer, then Eqs. (2.26) and (2.27) give

$$
\begin{aligned}
& I_{2 m+1}^{(2 q)}(0,0, \ldots, 0)= \frac{1}{m+1} \Gamma\left[\frac{2 q+1}{2 m+2}\right] \\
& \quad \times \exp \left[i \frac{\pi}{2}\left[\frac{2 q+1}{2 m+2}+2 q\right]\right], \\
& m=1,2, \ldots, q=0,1, . ., m .
\end{aligned}
$$

An alternative way of deriving Eqs. (2.29)-(2.31) is from the exact series representation of $I_{n}\left(a_{1}, a_{2}, \ldots, a_{n-1}\right)$ obtained in Ref. 2.

\section{EXAMPLES}

In this section we consider in more detail the cases $n=2$ (fold), $n=3$ (cusp), $n=4$ (swallowtail), and $n=5$ (butterfly). We verify that Eq. (2.19) is equivalent to known results for $n=2,3,4$. The differential equations we derive for the butterfly canonical integral $(n=5)$ are new and have not been reported in the literature before. In order to write down explicitly the differential equations contained in Eq. (2.19) for a given value of $n$, we have written a simple symbolic algebraic computer program based on the FORMAT statements of FORTRAN.

\section{A. The fold canonical integral $/ 2\left(a_{1}\right)$}

We have $n=2$ so that $l=1, p=0$ and Eq. (2.19) becomes

$$
\frac{d^{2} I_{2}^{(0)}}{d a_{1}^{2}}-\frac{1}{3} a_{1} I_{2}^{(0)}=0 .
$$

The fold integral $I_{2}\left(a_{1}\right)$ is related to the Airy integral Ai $\left(a_{1}\right)$ by

$$
I_{2}\left(a_{1}\right)=\left(2 \pi / 3^{1 / 3}\right) \mathrm{Ai}\left(a_{1} / 3^{1 / 3}\right),
$$

and if we set $a_{1}=x$, Eq. (3.1) becomes

$$
\frac{d^{2} \operatorname{Ai}(x)}{d x^{2}}-x \operatorname{Ai}(x)=0,
$$

which is a well-known result. ${ }^{15}$

\section{B. The cusp canonical integral $/ /_{3}\left(a_{1}, a_{2}\right)$}

In this case $n=3$ and either $l=2, p=0,1$ with $a_{1}=0$ or $l=1, p=0$. The differential equations obtained from Eq. (2.19) are for $l=2, p=0$

$$
\frac{\partial I_{3}^{(1)}}{\partial a_{2}}+i \frac{1}{2} a_{2} I_{3}^{(1)}=0, \quad a_{1}=0
$$

for $l=2, p=1$,

$$
\frac{\partial^{2} I_{3}^{(0)}}{\partial a_{2}^{2}}+i \frac{1}{2} a_{2} \frac{\partial I_{3}^{(0)}}{\partial a_{2}}+i \frac{1}{4} I_{3}^{(0)}=0, \quad a_{1}=0
$$

and for $l=1, p=0$

$$
\frac{\partial^{3} I_{3}^{(0)}}{\partial a_{1}{ }^{3}}-i \frac{1}{4} a_{1} I_{3}^{(0)}-\frac{1}{2} a_{2} \frac{\partial I_{3}^{(0)}}{\partial a_{1}}=0 .
$$

The cusp canonical integral $I_{3}\left(a_{1}, a_{2}\right)$ is the same as Pearcey's integral $P(x, y)$ provided we set $x=a_{2}, y=a_{1}$ and Eqs. (3.2)(3.4) are identical with Eqs. (2.12), (2.13), and (2.3), respectively, of Ref. 8.

Note that Eqs. (3.2) and (3.3) for $I_{3}^{(1)}$ and $I_{3}^{(0)}$ are not coupled together. Because of this we can solve Eq. (3.2) for the boundary condition $\partial P(0, y) / \partial y=0$ at $y=0$ [see Eq. (2.30)] obtaining

$$
\left.\frac{\partial P(x, y)}{\partial y}\right|_{y=0}=0 \text {. }
$$

This result also follows immediately from Eq. (1.1). Equation (3.5) is one of the initial conditions required for Eq. (3.4). The two remaining initial conditions can also be obtained explicitly from Eq. (3.3) in terms of Bessel functions of orders $\pm \frac{1}{4}$ and $\pm \frac{3}{4}$ and argument $x^{2} / 8$ [see Eqs. (5) and (8) of Ref. 5].

\section{The swallowtail canonical integral $I_{4}\left(a_{1}, a_{2}, a_{3}\right)$}

When $n=4$, we have the following possibilities

$$
\begin{array}{ll}
l=3, & p=0,1,2 \quad \text { with } a_{1}=a_{2}=0, \\
l=2, & p=0,1 \quad \text { with } a_{1}=0, \\
l=1, & p=0 .
\end{array}
$$

Equation (2.19) then yields the following differential equations: for $l=3$

$$
\left.\begin{array}{l}
(p=0) \quad \frac{\partial I_{4}^{(1)}}{\partial a_{3}}+\frac{3}{5} a_{3} I_{4}^{(2)}=0, \\
(p=1) \quad \frac{\partial I_{4}^{(2)}}{\partial a_{3}}-\frac{3}{5} a_{3} \frac{\partial I_{4}^{(0)}}{\partial a_{3}}-\frac{1}{5} I_{4}^{(0)}=0, \\
(p=2) \quad \frac{\partial^{2} I_{4}^{(0)}}{\partial a_{3}{ }^{2}}+\frac{3}{5} a_{3} \frac{\partial I_{4}^{(1)}}{\partial a_{3}}+\frac{2}{5} I_{4}^{(1)}=0,
\end{array}\right\} a_{1}=a_{2}=0,
$$


for $l=2$

$$
\left.\begin{array}{l}
(p=0) \frac{\partial^{2} I_{4}^{(0)}}{\partial a_{2}{ }^{2}}+i \frac{2}{5} a_{2} I_{4}^{(1)}+i \frac{3}{5} a_{3} \frac{\partial I_{4}^{(0)}}{\partial a_{2}}=0, \\
(p=1) \frac{\partial^{2} I_{4}^{(1)}}{\partial a_{2}{ }^{2}}-\frac{2}{5} a_{2} \frac{\partial I_{4}^{(0)}}{\partial a_{2}}+i \frac{3}{5} a_{3} \frac{\partial I_{4}^{(1)}}{\partial a_{2}}-\frac{1}{5} I_{4}^{(0)}=0,
\end{array}\right\} a_{1}=0,
$$

and for $l=1, p=0$

$$
\frac{\partial^{4} I_{4}^{(0)}}{\partial a_{1}{ }^{4}}+\frac{1}{5} a_{1} I_{4}^{(0)}-i \frac{2}{5} a_{2} \frac{\partial I_{4}^{(0)}}{\partial a_{1}}-\frac{3}{5} a_{3} \frac{\partial^{2} I_{4}^{(0)}}{\partial a_{1}{ }^{2}}=0 .
$$

In previous work ${ }^{8,9,27}$ the swallowtail canonical integral has been denoted $S(x, y, z)$ so we must make the correspondences $a_{1}=z, a_{2}=y, a_{3}=x$. Equations (3.6)-(3.8) are equivalent to those reported in Secs. 3.2-3.5 of Ref. 8 if it is also noted that $H=I_{4}^{(1)}$ and $G=I_{4}^{(2)}$.

\section{The butterfly canonical integral $/ 5\left(a_{1}, a_{2}, a_{3}, a_{4}\right)$}

We now have $n=5$ and the possible values for $l$ and $p$ are

$$
\begin{array}{ll}
l=4, \quad p=0,1,2,3 & \text { with } a_{1}=a_{2}=a_{3}=0, \\
l=3, \quad p=0,1,2 & \text { with } a_{1}=a_{2}=0, \\
l=2, \quad p=0,1 & \text { with } a_{1}=0 \\
l=1, & p=0 .
\end{array}
$$

From Eq. (2.19) we can obtain the following sets of differential equations: for $l=4$

$$
\left.\begin{array}{l}
(p=0) \frac{\partial I_{5}^{(1)}}{\partial a_{4}}-i \frac{2}{3} a_{4} I_{5}^{(3)}=0 \\
(p=1) \frac{\partial I_{5}^{(2)}}{\partial a_{4}}-\frac{2}{3} a_{4} \frac{\partial I_{5}^{(0)}}{\partial a_{4}}-\frac{1}{6} I_{5}^{(0)}=0, \\
(p=2) \frac{\partial I_{5}^{(3)}}{\partial a_{4}}-\frac{2}{3} a_{4} \frac{\partial I_{5}^{(1)}}{\partial a_{4}}-\frac{1}{3} I_{5}^{(1)}=0, \\
(p=3) \frac{\partial^{2} I_{5}^{(0)}}{\partial a_{4}^{2}}-i \frac{2}{3} a_{4} \frac{\partial I_{5}^{(2)}}{\partial a_{4}}-i \frac{1}{2} I_{5}^{(2)}=0,
\end{array}\right\} a_{1}=a_{2}=a_{3}=0
$$

for $l=3$

$$
\left.\begin{array}{l}
(p=0) \frac{\partial I_{5}^{(2)}}{\partial a_{3}}+i \frac{1}{2} a_{3} I_{5}^{(2)}-\frac{2}{3} a_{4} \frac{\partial I_{5}^{(0)}}{\partial a_{3}}=0 \\
(p=1) \frac{\partial^{2} I_{5}^{(0)}}{\partial a_{3}^{2}}+i \frac{1}{2} a_{3} \frac{\partial I_{5}^{(0)}}{\partial a_{3}}+\frac{2}{3} a_{4} \frac{\partial I_{5}^{(1)}}{\partial a_{3}}+i \frac{1}{6} I_{5}^{(0)}=0, \\
(p=2) \frac{\partial^{2} I_{5}^{(1)}}{\partial a_{3}^{2}}+i \frac{1}{2} a_{3} \frac{\partial I_{5}^{(1)}}{\partial a_{3}}+\frac{2}{3} a_{4} \frac{\partial I_{5}^{(2)}}{\partial a_{3}}+i \frac{1}{3} I_{5}^{(1)}=0,
\end{array}\right\} a_{1}=a_{2}=0,
$$

for $l=2$

$$
\begin{aligned}
& (p=0) \frac{\partial^{2} I_{s}^{(1)}}{\partial a_{2}{ }^{2}}-\frac{1}{3} a_{2} I_{5}^{(1)}-\frac{1}{2} a_{3} \frac{\partial I_{5}^{(0)}}{\partial a_{2}}+i \frac{2}{3} a_{4} \frac{\partial I_{s}^{(1)}}{\partial a_{2}}=0, \\
& (p=1) \frac{\partial^{3} I_{5}^{(0)}}{\partial a_{2}{ }^{3}}-\frac{1}{3} a_{2} \frac{\partial I_{s}^{(0)}}{\partial a_{2}}+i \frac{1}{2} a_{3} \frac{\partial I_{s}^{(1)}}{\partial a_{2}}+i \frac{2}{3} a_{4} \frac{\partial^{2} I_{s}^{(0)}}{\partial a_{2}{ }^{2}}-\frac{1}{6} I_{5}^{(0)}=0
\end{aligned}\left\{\begin{array}{l}
a_{1}=0 \\
a_{1}
\end{array}\right.
$$


and for $l=1, p=0$

$$
\begin{aligned}
& \frac{\partial^{5} I_{5}^{(0)}}{\partial a_{1}{ }^{5}}+i \frac{1}{6} a_{1} I_{5}^{(0)}+\frac{1}{3} a_{2} \frac{\partial I_{5}^{(0)}}{\partial a_{1}} \\
& -i \frac{1}{2} a_{3} \frac{\partial^{2} I_{5}^{(0)}}{\partial a_{1}{ }^{2}}-\frac{2}{3} a_{4} \frac{\partial^{3} I_{5}^{(0)}}{\partial a_{1}{ }^{3}}=0 .
\end{aligned}
$$

Equations (3.9)-(3.12) are new results for the butterfly canonical integral.

\section{UNIFORM ASYMPTOTIC CUSPOID APPROXIMATION FOR OSCILLATING INTEGRALS}

An important use of $I_{n}$ and its derivatives $\partial I_{n} / \partial a_{1}$, $\partial I_{n} / \partial a_{2}, \ldots, \partial I_{n} / \partial a_{n-1}$ is in the derivation of uniform asymptotic expansions ${ }^{1,2,10,19-23}$ for integrals of the form

$$
\int_{-\infty}^{\infty} g(t) \exp [i f(\alpha ; t) / \hbar] d t, \quad \hbar \rightarrow 0,
$$

where $f(\alpha ; t)$ is assumed to be real for real values of $t$ and to have $n$ coalescing real or complex saddle points defined by

$$
\frac{\partial f(\boldsymbol{\alpha} ; t)}{\partial t}=0 \quad \text { for } t=t_{j}(\boldsymbol{\alpha}), \quad j=1,2, \ldots, n \text {. }
$$

Note that the positions of the saddle points depend on a set of parameters $\boldsymbol{\alpha}$.

A key step in the analysis is a local one-to-one uniformly analytic change of variables $t \rightarrow u(\alpha ; t)$ defined by ${ }^{1,2,10,19-23}$

$$
f(\alpha ; t)=u^{n+1}+\sum_{i=1}^{n-1} a_{n-i} u^{n-i}+A,
$$

where the new parameters $\left\{a_{n-i}\right\}$ and $A$ depend on $\alpha$ but not on $t$.

In practical applications of uniform approximations, it is necessary to devise methods for calculating $\left\{a_{n-i}\right\}$ and $A$. The equations determining these quantities are $\mathrm{e}^{1,2,10,19-23}$

$$
f_{j}=u_{j}^{n+1}+\sum_{i=1}^{n-1} a_{n-i} u_{j}^{n-i}+A, j=1,2, \ldots, n,
$$

where the $u_{j}$ satisfy

$(n+1) u_{j}^{n}+\sum_{i=1}^{n-1}(n-i) a_{n-i} u_{j}^{n-i-1}=0, \quad j=1,2, \ldots, n$

and for notational convenience we have written $f_{j}=f\left(\alpha ; t_{j}\right)$.

For $n=2$ (fold) and $n=3$ (cusp), explicit formulas exist for the new parameters ${ }^{4,28-30}$ in terms of the $\left\{f_{j}\right\}$. For $n \geqslant 4$, however, this is no longer true. To overcome this problem for the swallowtail case $n=4$, we have developed iterative and algebraic techniques for the calculation of the parameters. ${ }^{9}$ In this section we outline how these two techniques can be generalized from the case $n=4$ to arbitrary values of $n$, thereby allowing the application of uniform asymptotic techniques to oscillating integrals with an arbitrary number of coalescing saddle points.

\section{A. Iterative method}

The iterative method $^{9}$ is applicable when the $\left\{a_{n-i}\right\}$ are not close to the caustic associated with the transformation (4.3). On the caustic surface, two or more of the $t_{j}$ (and hence also the $f_{j}$ and $u_{j}$ ) have coalesced.
First we simplify Eq. (4.4). Multiplying Eq. (4.5) by $u_{j}$ shows that

$$
u_{j}^{n+1}=-(u+1)^{-1} \sum_{i=1}^{n-1}(n-i) a_{n-i} u_{j}^{n-i}, j=1,2, \ldots, n \text {, }
$$

and substituting Eq. (4.6) into Eq. (4.4) gives

$$
f_{j}=\sum_{i=1}^{n-1} \frac{i+1}{n+1} a_{n-i} u_{j}^{n-i}+A, j=1,2, \ldots, n .
$$

The $t_{j}$ and $u_{j}$ are either purely real or occur as complex conjugate pairs and it is important to consider how they are to be ordered. We order the $t_{j}$ and $u_{j}$ so that all complex conjugate pairs are next to each other and are before any purely real $t_{j}$ and $u_{j}$. Next we construct the quantities

$$
\begin{aligned}
& \sum_{i=1}^{n-1} \frac{i+1}{n+1} a_{n-i}\left(u_{2 k-1}^{n-i}-u_{2 k}^{n-i}\right)=f_{2 k-1}-f_{2 k}, \\
& k=1,2, \ldots, \operatorname{int}(n / 2)
\end{aligned}
$$

and

$$
\begin{aligned}
& \sum_{i=1}^{n-1} \frac{i+1}{n+1} a_{n-i}\left(u_{2 k-1}^{n-i}+u_{2 k}^{n-i}-u_{2 k+1}^{n-i}-u_{2 k+2}^{n-i}\right) \\
& =f_{2 k-1}+f_{2 k}-f_{2 k+1}-f_{2 k+2} \\
& k=1,2, \ldots, \operatorname{int}(n / 2)-1
\end{aligned}
$$

where as before int = integer part of. When $n$ is an odd integer, we also need the extra equation

$$
\begin{gathered}
\sum_{i=1}^{n-1} \frac{i+1}{n+1} a_{n-i}\left[\frac{1}{2}\left(u_{n-2}^{n-i}+u_{n-1}^{n-i}\right)-u_{n}^{n-i}\right] \\
=\frac{1}{2}\left(f_{n-2}+f_{n-1}\right)-f_{n} .
\end{gathered}
$$

Note there is always one real root of Eq. (4.5) when $n$ is odd and in Eq. (4.10) we label this root to be $u_{n}$.

The left- and right-hand sides of Eqs. (4.8)-(4.10) are both either real or purely imaginary and so can always be written in a purely real form. For given values of $\left\{u_{j}\right\}$ and $\left\{f_{i}\right\}$, Eqs. $(4.8)-(4.10)$ represent $n-1$ linear equations in the $n-1$ variables $a_{n-i}$ with $i=1,2, \ldots, n-1$. The iterative procedure to solve them is as follows

(a) Make an initial guess for the vector $\mathbf{a}^{(0)}$ $=\left(a_{1}^{(0)}, a_{2}^{(0)}, \ldots, a_{n-1}^{(0)}\right)$.

(b) Solve the polynomial Eq. (4.5) for its roots $u_{j}\left(\mathbf{a}^{0}\right)$ $j=1,2, \ldots, n$.

(c) Order the $u_{j}$ in the same way as the saddle points $t_{j}$ of the function $f(\alpha ; t)$, i.e., we have $u=u_{j} \leftrightarrow t=t_{j}$ for $j=1,2, \ldots, n$.

(d) Solve the linear set of Eqs. (4.8)-(4.10) to obtain a new value for the vector of coefficients $\mathbf{a}^{(1)}$.

(e) Return to (b) and iterate until convergence is achieved.

(f) The remaining coefficient $A$ can then be obtained from Eq. (4.7).

Notice this iterative technique fails if two or more saddle points have coalesced, i.e., if they are on the caustic. In practice it is expected that the method will also become ill conditioned as the $\left\{a_{n-i}\right\}$ approach the caustic. 


\section{B. Algebraic method}

Unlike the iterative scheme, the algebraic method ${ }^{4,9,30}$ is expected to be most useful for $\left\{a_{n-i}\right\}$ actually on the caustic. The basic idea is to obtain a set of simultaneous polynomial equations for the $\left\{a_{n-i}\right\}$. $\mathrm{als}^{31}$

First, we define the elementary symmetric polynomi-

$$
\begin{aligned}
& \tau_{1}\left\{r_{i}\right\}=\sum_{i=1}^{n} r_{i}, \\
& \tau_{2}\left\{r_{i}\right\}=\sum_{i>j}^{n} \sum_{j=1}^{n} r_{i} r_{j}, \\
& \tau_{3}\left\{r_{i}\right\}=\sum_{i>j}^{n} \sum_{j>k}^{n} \sum_{k=1}^{n} r_{i} r_{j} r_{k}, \\
& \vdots \vdots \\
& \tau_{n}\left\{r_{i}\right\}=r_{1} r_{2} r_{3} \cdots r_{n},
\end{aligned}
$$

together with

$$
s_{m}\left\{r_{i}\right\}=\sum_{i=1}^{n} r_{i}^{m} \quad m=1,2, \ldots .
$$

Second, we introduce the following symmetric functions of the $\left\{f_{i}\right\}$

$$
\begin{gathered}
\bar{f}_{1}=\left(\begin{array}{l}
n \\
1
\end{array}\right)^{-1} \tau_{1}\left\{f_{i}\right\}, \\
\bar{f}_{2}=\left(\begin{array}{l}
n \\
2
\end{array}\right)^{-1} \tau_{2}\left\{f_{i}-\bar{f}_{1}\right\}, \\
\bar{f}_{3}=\left(\begin{array}{l}
n \\
3
\end{array}\right)^{-1} \tau_{3}\left\{f_{i}-\bar{f}_{1}\right\}, \\
\vdots \vdots \\
\bar{f}_{j}=\left(\begin{array}{l}
n \\
j
\end{array}\right)^{-1} \tau_{j}\left\{f_{i}-\bar{f}_{1}\right\}, \\
\vdots \vdots \\
\bar{f}_{n}=\left(\begin{array}{l}
n \\
n
\end{array}\right)^{-1} \tau_{n}\left\{f_{i}-\bar{f}_{1}\right\},
\end{gathered}
$$

where the $\left(\begin{array}{l}n \\ j\end{array}\right)$ are binomial coefficients. Notice that the $\bar{f}_{j}$ are all real even if some of the $f_{i}$ are complex. Since everything on the right-hand sides of Eq. (4.13) is assumed to be known, the $\left\{\bar{f}_{j}\right\}$ are also known quantities. We now write each $\tau_{j}\left\{f_{i}-\bar{f}\right\}$ in terms of $\left\{\tau_{j}\left\{f_{i}\right\}\right\}$ and then in terms of $\left\{s_{j}\left\{f_{i}\right\}\right\}$. We now use Eq. (4.7) to express the $s_{i}\left\{f_{i}\right\}$ in terms of $\left\{s_{m}\left\{u_{i}\right\}\right\}$. At this point we have obtained $\left\{f_{j}\right\}$ in terms of $\left\{s_{m}\left\{u_{i}\right\}\right\}$. The final step is to use Newton's formulas ${ }^{32}$ for the sums of powers of the roots of the polynomial Eq. (4.5) to write $\left\{s_{m}\left\{u_{i}\right\}\right\}$ and hence, $\left\{\bar{f}_{j}\right\}$ in terms of $\left\{a_{n-i}\right\}$. It should be noted that the algebraic steps just described involve a huge amount of manipulation for $n \geqslant 4$ and it is only feasible to carry them out with the help of a symbolic algebraic computer program such as REDUCE or MACSYMA.

The result of the operations outlined above is a set of $n-1$ polynomial equations in the variables $a_{n-i}$, $i=1,2, \ldots, n-1$ and one equation in the $\left\{a_{n-i}\right\}$ and $A$. The solution of these equations for given input data $\left\{f_{j}\right\}$ then yields the desired values of $\left\{a_{n-i}\right\}$ and $A$.

The main advantage of the algebraic method is that it remains valid on the caustic. Indeed in this circumstance the set of polynomial equations for the $\left\{a_{n-i}\right\}$ and $A$ are expected to be easier to solve numerically than when the $\left\{a_{n-i}\right\}$ are off the caustic. In addition, if $f(\alpha ; t)$ has a special symmetry which results in some of the $a_{n-i}$ being identically zero, the set of polynomial equations will simplify and also be easier to solve. Thus in practice the iterative and algebraic methods for determining $\left\{a_{n-i}\right\}$ and $A$ are expected to be complementary.

\section{SUMMARY AND CONCLUSIONS}

In this paper we have derived the differential equations satisfied by the cuspoid canonical integral $I_{n}(\mathbf{a})$ and its partial derivatives for any value of $n$. Our method consists in finding a set of coupled linear ordinary differential equations for each step in the sequence $I_{n}(0,0, \ldots, 0,0)$ $\rightarrow I_{n}\left(0,0, \ldots, 0, a_{n-1}\right) \rightarrow I_{n}\left(0,0, \ldots, a_{n-2}, a_{n-1}\right)$ $\rightarrow \cdots \rightarrow I_{n}\left(0, a_{2}, \ldots, a_{n-2}, a_{n-1}\right) \rightarrow I_{n}\left(a_{1}, a_{2}, \ldots, a_{n-2}, a_{n-1}\right)$. The results obtained in Sec. II provide everything that is required for the method (differential equations, transformations, and initial conditions).

In practical implementations of this technique for the numerical evaluation of $I_{n}(\mathbf{a})$ and its partial derivatives, it is also necessary to consider the stability of the differential equations when they are integrated. Previous calculations for $n=3$ (cusp) $)^{4-6,18}$ and $n=4$ (swallowtail) ${ }^{8}$ indicate that the method will be numerically stable when all the saddle points of $P_{n}(u)$ [see Eq. (2.2)] are real, because this condition implies that the independent solutions of the differential equations are then all of oscillatory type. It is important to note that the region of $\left(a_{1}, a_{2}, \ldots, a_{n-1}\right)$ space which corresponds to all real saddle points for $P_{n}(u)$ is also the region where $I_{n}$ (a) has the richest structure. Outside of this region the independent solutions of the differential equations will contain exponentially increasing solutions which will eventually limit the accuracy to which $I_{n}(\mathbf{a})$ can be computed.

We have also considered the problem of determining the parameters a for use in the uniform asymptotic cuspoid approximation. We have shown how the iterative and algebraic methods developed earlier ${ }^{9}$ for the special case of $n=4$ can be generalized to arbitrary values of $n$.

Finally, we wish to emphasize that the treatment presented in this paper has unified and generalized previous researches on the cuspoid canonical integrals, in which each value of $n$ had been treated as a special case.

\section{ACKNOWLEDGMENTS}

J.N.L.C. thanks Professor R. A. Marcus for his hospitality at the A.A. Noyes Laboratory of Chemical Physics, California Institute of Technology, Pasadena, California and NATO for a Senior Scientist award. P.R.C. thanks the States of Jersey Education Committee for a research studentship. The algebraic calculations were carried out on the CDC 7600 computer of the University of Manchester Regional Computer Centre. 
${ }^{1}$ J. N. L. Connor, Mol. Phys. 26, 1217 (1973).

${ }^{2}$ J. N. L. Connor, Mol. Phys. 27, 853 (1974). On p. 861, lines 25 and 26, the words "even" and "odd" should be interchanged.

${ }^{3}$ F. J. Wright, J. Phys. A: Math. Gen. 13, 2913 (1980).

${ }^{4}$ J. N. L. Connor and D. Farrelly, J. Chem. Phys. 75, 2831 (1981).

5J. N. L. Connor and D. Farrelly, Chem. Phys. Lett. 81, 306 (1981).

${ }^{6}$ J. N. L. Connor and P. R. Curtis, J. Phys. A: Math. Gen. 15, 1179 (1982).

${ }^{7}$ J. J. Stamnes and B. Spjelkavik, Opt. Acta 30, 1331 (1983).

${ }^{8}$ J. N. L. Connor, P. R. Curtis, and D. Farrelly, Mol. Phys. 48, 1305 (1983). In Eq. (3.46) for $\frac{4}{3} \Gamma\left(\frac{3}{3}\right) \operatorname{read} \frac{3}{3} \Gamma\left(\frac{3}{3}\right)$.

${ }^{9}$ J. N. L. Connor, P. R. Curtis, and D. Farrelly, J. Phys. A: Math. Gen. 17, 283 (1984). In Table 4, for $c=-20.957$ read $c=-20.597$.

${ }^{10}$ J. N. L. Connor, Mol. Phys. 31, 33 (1976).

"R. Thom, Structural Stability and Morphogenesis. An Outline of a General Theory of Models, translated from the French edition, as updated by the author, by D. H. Fowler, with a Foreword by C. H. Waddington (Benjamin, Reading, MA, 1975).

${ }^{12} \mathrm{~T}$. Poston and I. Stewart, Catastrophe Theory and its Applications (Pitman, London, 1978).

${ }^{13}$ R. Gilmore, Catastrophe Theory for Scientists and Engineers (Wiley, New York, 1981)

${ }^{14}$ G. B. Airy, Trans. Cambridge Philos. Soc. 6, 379 (1838).

${ }^{15} \mathrm{~J}$. C. P. Miller, The Airy Integral, Giving Tables of Solutions of the Differential Equation $y^{\prime \prime}=x y$ (Cambridge U.P., Cambridge, England, 1971), Part-Vol. B.

${ }^{16}$ L. Brillouin, Ann. Sci. École Norm. Sup., 3rd Ser. 33, 17 (1916).

${ }^{17}$ T. Pearcey, Philos. Mag. 37, 311 (1946).
${ }^{18}$ T. Pearcey and G. W. Hill, editors, The Effect of Aberrations Upon the Optical Focus (Commonwealth, Scientific and Industrial Research Organization, Melbourne, Australia, 1963).

${ }^{19}$ D. Ludwig, Commun. Pure Appl. Math. 19, 215 (1966).

${ }^{20}$ N. Bleistein, J. Math. Mech. 17, 533 (1967).

${ }^{21}$ S. O. Rice, Bell Syst. Tech. J. 47, 1970 (1968).

${ }^{22}$ F. Ursell, Proc. Cambridge Philos. Soc. 72, 49 (1972).

${ }^{23} J$. Martin, Proc. Cambridge Philos. Soc. 76, 211 (1974).

${ }^{24}$ G. H. Hardy, Quart. J. 41, 226 (1910), reprinted in Collected Papers of G. H. Hardy, Vol. IV, edited by a Committee appointed by The London Mathematical Society (Clarendon, Oxford, 1969), p. 460.

${ }^{25}$ C. A. Stewart, Advanced Calculus (Methuen, London, 1951), 3rd ed., Sec. 12.7 .

${ }^{26} \mathrm{~W}$. Gröbner and N. Hofreiter, Integraltafel, Zweiter Teil Bestimmte Integrale, Funfte, verbesserte Auflage (Springer, Vienna, Austria, 1973), Sec. 333 , p. 118 , items (11a) and (11b).

${ }^{27}$ J. N. L. Connor, P. R. Curtis, C. J. Edge, and A. Laganà, J. Chem. Phys. 80, 1362 (1984).

${ }^{28}$ C. Chester, B. Friedman, and F. Ursell, Proc. Cambridge Philos. Soc. 53, $599(1957)$.

${ }^{29}$ J. N. L. Connor and R. A. Marcus, J. Chem. Phys. 55, 5636 (1971).

${ }^{30}$ D. H. Peregrine and R. Smith, Philos. Trans. R. Soc. London, Ser. A 292, 341 (1979).

${ }^{31}$ A. Mostowski and M. Stark, Introduction to Higher Algebra, translated from the Polish by J. Musielak (Pergamon, Oxford, 1964), p. 346.

${ }^{32} \mathrm{~J}$. V. Uspensky, Theory of Equations (McGraw-Hill, New York, 1948), pp. 260-262. 